\title{
Long-term outcome of surgical excision for treatment of cervicofacial granulomatous lymphadenitis in children
}

\author{
Quentin Neven $^{1} \cdot$ Dimitri Van der Linden ${ }^{1,2} \cdot$ Marc Hainaut $^{3} \cdot$ Sandra Schmitz $^{2,4}$
}

Received: 18 December 2019 / Accepted: 21 February 2020 / Published online: 6 March 2020

(c) Springer-Verlag GmbH Germany, part of Springer Nature 2020

\begin{abstract}
Purpose Granulomatous inflammation is a common cause of subacute cervicofacial lymphadenitis in children. Nontuberculous mycobacterial (NTM) infections and cat-scratch disease (CSD) are the most frequent causes. Optimal treatment, which may include surgery, antibiotic treatment or wait-and-see approach, is debatable. The goal of this study was to compare the short- and long-term outcome of various surgical procedures.

Methods Case series with a chart review of all children treated by surgical excision of granulomatous lymph nodes in the cervicofacial area from 2000 to 2016 at two tertiary care centers.

Results Forty patients were included in this study. The median age at first symptoms was 3.7 years (13 months-14 years). Mean follow-up was 5.8 years (6 months-15.3 years). 25 patients fit with diagnosis of NTM infection, 6 with CSD while diagnosis remained uncertain in 9 patients. The primary surgical procedure consisted of total excision $(n=27)$, incision/ drainage $(n=9)$ or incomplete excision $(n=4)$. None of the patients treated by primary complete excision needed further intervention contrary to the group of patients with incomplete surgical procedures where additional surgical management was required in 54\%. At follow-up, all patients were healthy without evidence of recurrence.

Conclusion We advocate early surgical intervention with complete excision to reach quick resolution and reduce the need for additional surgery. The long-term outcome was favorable.
\end{abstract}

Keywords Children $\cdot$ Lymphadenitis $\cdot$ Nontuberculous mycobacteria $\cdot$ Cat-scratch disease $\cdot$ Surgery

\section{Abbreviations}

BH Bartonella henselae

CSD Cat-scratch disease

CSDL Cat-scratch disease lymphadenitis

FNA Fine-needle aspiration

NTM Nontuberculous mycobacteria

NTML Nontuberculous mycobacteria lymphadenitis

PCR Polymerase chain reaction

TST Tuberculin skin testing

Sandra Schmitz

sandra.schmitz@uclouvain.be

1 Pediatric Infectious Diseases-General Pediatrics, St Luc University Hospital, Brussels, Belgium

2 Institute of Experimental and Clinical Research (IREC), Catholic University of Louvain, Brussels, Belgium

3 Department of Pediatrics, CHU Saint-Pierre, Brussels, Belgium

$4 \quad$ Head and Neck Surgery Unit, Institut Roi Albert II-St Luc University Hospital, Avenue Hippocrate 10, 1200 Brussels, Belgium
US Unspecified

IGRA Interferon-gamma release assay

CRP C-reactive protein

LDH Lactate dehydrogenase

\section{Introduction}

Enlarged lymph nodes are a common symptom in a number of infectious, autoimmune and malignant diseases in childhood. After reactive hyperplasia, granulomatous inflammation is the second most common finding in histological examinations of cervicofacial lymph nodes, regardless of the age of the child [1]. Granuloma formation is a protective response of our lymph nodes induced by the presence of substances, such as some microorganisms or foreign bodies, that are difficult to eliminate by the usual process of phagocytosis by macrophages [2]. Modified macrophages (epithelioid cells) aggregate to form granulomas surrounded by lymphocytes. Granulomas are further classified histologically by the presence or absence of 
caseous necrosis. Among infectious causes, nontuberculous mycobacteria (NTM) and cat-scratch disease (CSD) seem to be the predominant etiologies in children [1].

Clinical and histopathological characteristics of cervicofacial CSD lymphadenitis (CSDL) are similar to those of NTM lymphadenitis (NTML) [3]. Children with both types of infections have painless unilateral neck node(s), typically in the submandibular or preauricular areas, who do not respond to standard antibiotics. Bilateral enlarged lymph nodes are uncommon. Over a period of weeks or months and in the absence of effective therapy, the lesions usually progress to liquefaction with erythema or purplish color changes of the overlying skin. Fistula formation can occur followed by a spontaneous drainage through the skin (Fig. 1) [4]. Even if the disease remains typically localized to the clinically affected area, disseminated NTM and CSD infections are described $[5,6]$ and can be a manifestation of immune deficiency.

The management of granulomatous lymphadenitis is frequently based on the combination or sequential use of macrolides, antituberculous drugs and surgery. Various surgical procedures have been described with very different cure and complication rates. Indeed, while complete excision can be complicated by facial nerve paresis, a lesser procedure increases the risks of failure to heal, recurrence, reoperation and scar formation [7].

The aim of this study was to describe clinical, microbiological and histological findings of a series of patients with isolated cervical lymphadenopathies and determine the value of complete excision as a diagnostic and therapeutic tool in the short and long term.

\section{Materials and methods}

We performed a case series with a chart review of all children who underwent surgical excision of cervicofacial granulomatous lymph nodes from January 1, 2000 to

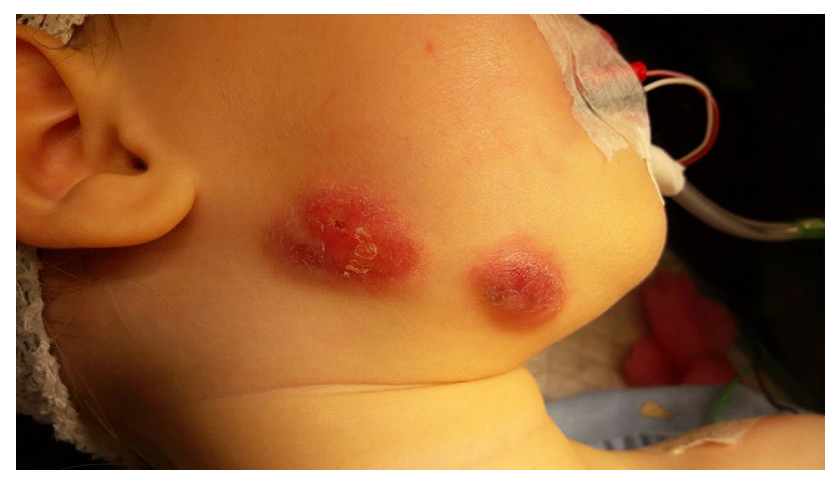

Fig. 1 NTML in the submandibular area with skin color change and draining cutaneous fistula
March 1, 2016 in two tertiary care centers in Brussels, Belgium.

Patients were included in the study if they met all the following inclusion criteria: (1) children aged 16 years or younger, (2) persistent head and neck mass for more than 4 weeks, (3) pathological findings of granulomatous inflammation on surgical specimens.

Immunocompromised patients and patients with disseminated infection were excluded.

Data were extracted from all electronic medical records and completed by calling or writing to parents and/or general practitioners and included the following information: age, gender, immune status, bacille Calmette-Guérin (BCG) vaccination, mode of presentation, general clinical aspect, site of infection, and the use of antibiotic treatment. Special attention was given to diagnostic procedures, surgical treatment, early outcome, and long-term follow-up. The primary endpoint was healing outcome. The secondary endpoint was long-term functional and esthetic outcome.

Patients were divided into three groups according to final diagnoses. Children with proven NTLM (NTM group) met one or more of the following criteria: (1) positive tuberculin skin test in the absence of BCG vaccination or previous medical history of mycobacterial disease, (2) positive mycobacterial cultures and/or positive polymerase chain reaction (PCR) for NTM and/or positive stains for acid fast bacilli (AFB) from fine-needle aspiration (FNA) or surgical specimens. Diagnosis of proven CSDL (CSD group) was made if one or more of the following criteria was present: (1) positive PCR assay for a bartonella species from FNA or surgical specimens, (2) IgM antibodies against Bartonella henselae titer $\geq 1: 100$ or IgG titer $\geq 1: 512$. The third group (unspecified-US) consisted of children without definitive diagnosis. Primary and secondary endpoints were compared between these three groups.

\section{Results}

\section{Demographic characteristics and clinical findings}

Forty patients were included in this case series. No patients had significant past medical history, except one boy who had been diagnosed with neuroblastoma 3 years before. No patients had clinical evidence of impaired immune function or had received an immunosuppressive drugs at time of clinical presentation. All patients looked healthy and none presented constitutional symptoms such as fever, recent weight loss or night sweats.

Twenty-five patients fit with diagnosis of NTM infection, 6 fit with that of CSD while diagnosis remained uncertain in 9 patients classified as the US group. The 
demographic and clinical data are presented in Table 1 . The median age of NTM group was 3.1 years (range, 15 months -8 years). A median age of 5.8 years (range, 13 months-14 years) was observed in patients infected with $B$. henselae. Two girls in the NTM group were born from consanguineous parents.

Clinical findings were similar within NTM and CSD groups. Cervical lymph nodes were enlarged predominantly in the submandibular area, near the angle of the jaw (68\%), measured greater than $2 \mathrm{~cm}$ in diameter, with fluctuation and color changes in 26 cases. Draining cutaneous fistula occurred in 15 cases (38\%), spontaneously in 8 cases or after FNA in 7 cases. Left and right side were indifferently affected. No significant difference regarding the side or the localization of enlarged lymph nodes was observed within one of the three groups. Only one patient infected with NTM presented bilateral disease (right anterior cervical and left submandibular areas). One patient with submandibular enlarged lymph nodes presented a Horner's syndrome, which resolved after complete excision.

The onset of disease for the NTM infections was noticed more often in the fall and winter months, with $65 \%$ of cases having occurred between November and February. All presentations of CSD infection were recorded in the spring and fall seasons.

\section{Initial treatment and course of the disease}

All patients were initially treated with various combinations of antibiotics for 5 days -6 months before referral without clear clinical benefits. 24 patients received few weeks of clarithromycin, alone $(n=18)$ or in addition to rifampicin $(n=6)$.

The median time between the onset of symptoms and the first surgical procedure was 2.7 months (range, 1-9 months). A higher proportion of children in the US group were referred during an early stage, before skin changes or lymph node fluctuation.

\section{Management and outcome}

A total of 49 surgical procedures under general anesthesia were performed in these 40 patients and are summarized in Table 2 . The primary surgical procedures included complete excision $(n=27)$, incision/drainage $(n=9)$, and incomplete
Table 1 Demographic and clinical findings

\begin{tabular}{|c|c|c|c|c|}
\hline & Total $n=40$ & NTM group $n=25$ & CSD group $n=6$ & US group $n=9$ \\
\hline \multicolumn{5}{|l|}{ Age at onset of disease, years } \\
\hline Median & 3.9 & 3.1 & 5.8 & 5.0 \\
\hline Range & $1.1-14$ & $1.2-8.1$ & $1.1-14.4$ & $1.8-13.7$ \\
\hline Sex ratio, (male/female) & 1.1 & 0.7 & 1.5 & 1.2 \\
\hline Consanguinity, $n(\%)$ & $2(5)$ & $2(8)$ & 0 & 0 \\
\hline General symptoms, $n(\%)$ & 0 & 0 & 0 & 0 \\
\hline \multicolumn{5}{|l|}{ Stage of lymphadenitis, $n(\%)$} \\
\hline Early, without skin discoloration & $14(35)$ & $7(28)$ & $2(33)$ & $5(56)$ \\
\hline Skin discoloration & $26(65)$ & $18(72)$ & $4(67)$ & $4(44)$ \\
\hline Draining cutaneous fistula, $n(\%)$ & $15(38)$ & $11(44)$ & $2(33)$ & $2(22)$ \\
\hline \multicolumn{5}{|l|}{ Diagnostic delay, months } \\
\hline Median & 2.7 & 2.6 & 2.8 & 3.1 \\
\hline Range & $1-9$ & $1-6$ & $1-9$ & $1-7$ \\
\hline \multicolumn{5}{|l|}{ Site, $n(\%)$} \\
\hline Submandibular & $27(68)$ & $18(72)$ & $5(83)$ & $4(44)$ \\
\hline Anterior cervical & $5(12)$ & $2(8)$ & 0 & $3(33)$ \\
\hline Parotid & $3(8)$ & $2(8)$ & 0 & $1(11)$ \\
\hline Submental & $3(8)$ & $2(8)$ & 0 & $1(11)$ \\
\hline Preauricular & $1(3)$ & $1(4)$ & 0 & 0 \\
\hline Posterior triangle & $1(3)$ & 0 & 0 & $1(11)$ \\
\hline Retroauricular & $1(3)$ & 0 & $1(17)$ & 0 \\
\hline Supraclavicular & 0 & 0 & 0 & 0 \\
\hline \multicolumn{5}{|l|}{ Side, $n(\%)$} \\
\hline Left & $20(50)$ & $12(48)$ & $3(50)$ & $5(56)$ \\
\hline Right & $19(48)$ & $12(48)$ & $3(50)$ & $4(44)$ \\
\hline Bilateral & $1(3)$ & $1(4)$ & 0 & 0 \\
\hline
\end{tabular}


excision $(n=4)$. No recurrence occurred in the group of patients having undergone initial complete excision surgery. Secondary complete excision for relapse or persistent draining fistula was required in five patients (56\%) having initially undergone incision/drainage and a third surgical procedure was performed in two individuals. The median time between the first two surgeries was 8.5 months (range, 18 days -25 months). Two patients out of four with initial incomplete excision required a secondary complete excision. Among the seven patients who relapsed after incomplete excision or incision/drainage, five were referred to our centers after primary unsuccessful surgical treatment performed in other hospitals.

Information about postoperative medical treatment was available for 36 patients. 16 out of these 36 patients (44\%) were treated either by azithromycin $(n=1)$, clarithromy$\operatorname{cin}(n=11)$ or by a combination of clarithromycin and rifampicin $(n=4)$. Antibiotic treatment was given for an average of 3 weeks (range 1-6 weeks) after primary surgery. Four patients ceased therapy because of side effects. The rationale for placing these patients on antibiotic therapy and the remaining children to just monitor depended on surgeon experience.
The mean time of hospitalization was 3.9 days (range, 2-14 days).

\section{Functional or esthetic complications and long-term follow-up}

All children $(n=40)$ were examined at the 2-week follow-up visit after surgery. Long-term follow-up data were available for 32 patients $(80 \%)$ with a median follow-up of 5.8 years (range $0.5-15.3$ years). Early postoperative functional weakness of the marginal branch of the facial nerve was observed in eight patients after complete resection of a lesion localized in the submandibular area. Nerve palsy was transient in six patients with complete resolution of symptoms within 12 weeks (range, 1-12 weeks), while two patients kept mild lip asymmetry when smiling. Implemented in our centers over last 2 years of the inclusion process, facial nerve monitoring was utilized in five patients who underwent primary complete excision and none of them had facial nerve damage, either transient or permanent. Consequently, the rate of permanent facial nerve palsy after complete excision was $2 / 29(7 \%)$ before and $0 / 5(0 \%)$ after initiation of nerve monitoring.
Table 2 Summary of treatment approaches, outcome and follow-up

\begin{tabular}{|c|c|c|c|c|}
\hline & Total & Complete excision & $\begin{array}{l}\text { Incomplete } \\
\text { excision }\end{array}$ & $\begin{array}{l}\text { Incision } \\
\text { and drain- } \\
\text { age }\end{array}$ \\
\hline First operation, $n$ & 40 & 27 & 4 & 9 \\
\hline $\begin{array}{l}\text { Postoperative antibiotic treatment after first } \\
\text { intervention (when data available), } n(\%)\end{array}$ & $16(44)$ & $10(37)$ & $2(67)$ & $4(67)$ \\
\hline Recurrence after first intervention, $n(\%)$ & $7(17)$ & 0 & $2(50)$ & $5(56)$ \\
\hline \multicolumn{5}{|l|}{ Time between 1e and $2 \mathrm{e}$ intervention, months } \\
\hline Median & 6.2 & NA & 0.6 & 8.5 \\
\hline Range & $0.5-25$ & NA & $0.5-0.6$ & $0.6-25$ \\
\hline \multicolumn{5}{|l|}{ Number of procedures per patient, $n(\%)$} \\
\hline 1 & & $27(100)$ & $2(50)$ & $4(44)$ \\
\hline 2 & & 0 & $2(50)$ & $3(33)$ \\
\hline 3 & & 0 & 0 & $2(22)$ \\
\hline \multicolumn{5}{|l|}{ Length of hospital stay, days } \\
\hline Median & 3.9 & 3.8 & 3.3 & 4.5 \\
\hline Range & $2-14$ & $2-14$ & $2-5$ & $2-10$ \\
\hline Last operation, $n$ & 40 & 34 & 2 & 4 \\
\hline \multicolumn{5}{|l|}{ Long-term follow-up } \\
\hline Data available, $n(\%)$ & $32(80)$ & $27(79)$ & $2(100)$ & $3(75)$ \\
\hline Median duration, years & 5.8 & 5.8 & 6.1 & 6.2 \\
\hline range & $0.5-15.3$ & $0.5-15.3$ & $3.9-8.3$ & $0.5-12.4$ \\
\hline Facial nerve paralysis, $n(\%)$ & $8(20)$ & $8(24)$ & 0 & 0 \\
\hline Transient & $6(15)$ & $6(18)$ & l & l \\
\hline Permanent & $2(5)$ & $2(6)$ & l & l \\
\hline \multicolumn{5}{|l|}{ Esthetic result } \\
\hline Keloid scar, $n(\%)$ & $4(10)$ & $3(9)$ & 0 & $1(25)$ \\
\hline
\end{tabular}




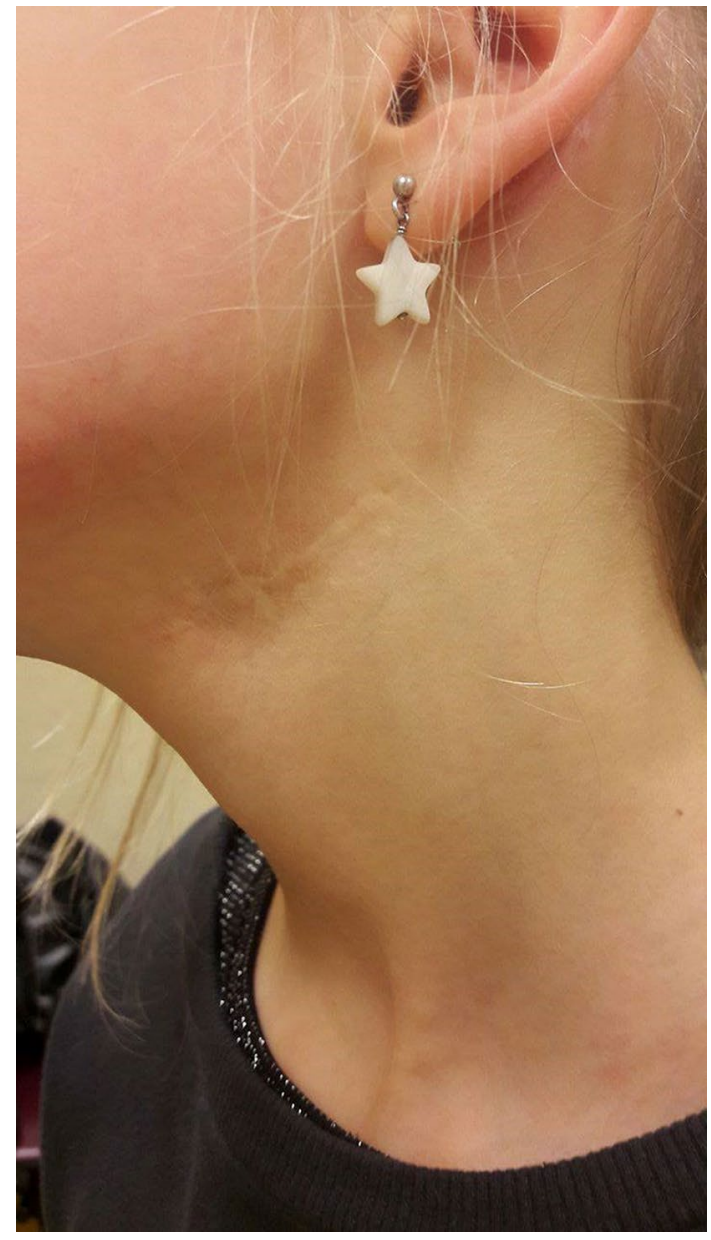

Fig. 2 10-year-old girl, treated by complete excision of NTML 6 years earlier
Four patients $(10 \%)$ developed keloids. Two of them underwent surgery and one received intralesional steroid injections with good esthetic results. Satisfactory wound healing was described in all other patients (Fig. 2).

At long-term follow-up, all patients were healthy without evidence of recurrence and none presented other mycobacterial or opportunistic infections.

\section{Histological findings}

Chronic granulomatous inflammation was associated with areas of necrosis in 32 cases ( $80 \%)$. Based on our retrospective analysis of pathology records, it is difficult to identify caseous necrosis within the samples. Langhans giant cells were frequently described but calcifications were detected in only three patient samples. Although performed unsystematically, Ziehl-Neelsen and auramine staining led to the detection of acid-fast bacilli in 2/19 and 3/7 cases, respectively. No Warthin-Starry silver staining was performed. In the majority of cases, tuberculosis could not be ruled out based on histological analysis alone.

\section{Laboratory data and tuberculin skin testing results}

The results of the diagnostic procedures are summarized in Table 3.

All patients displayed normal blood count. Seven out of 34 patients tested positive for $B$. henselae by immunofluorescence antibody test. No serological follow-up was performed on patients with an initial negative result.

Table 3 Diagnosis procedures performed

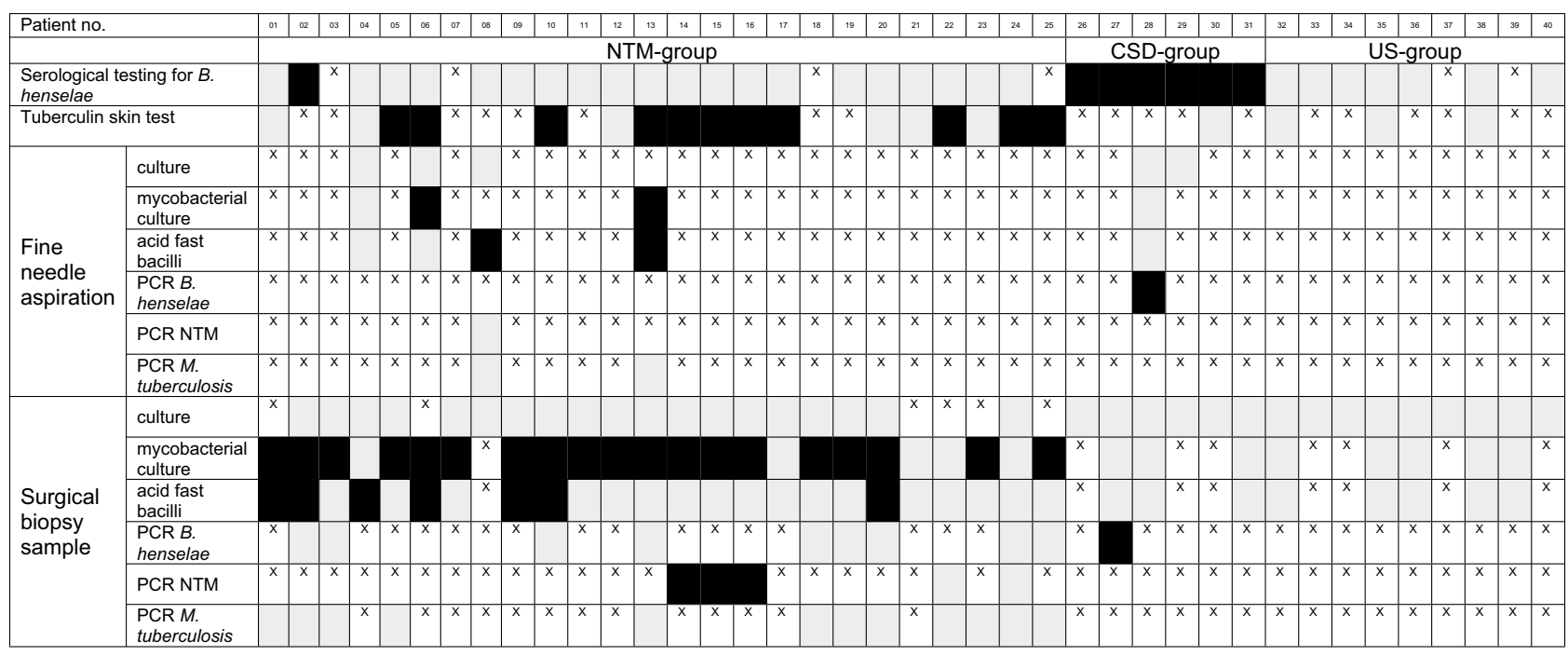

\begin{tabular}{|l|l|}
\hline $\mathrm{X}$ & procedure not performed \\
\hline & negative result \\
\hline positive result
\end{tabular} 
Tuberculin skin testing (TST) was performed on 21 children (53\%) and was reactive in 11 cases. Chest radiography performed in 23 children was unremarkable.

FNA was performed as an outpatient procedure in 14 patients. FNA results were acellular in 4 cases, showed nonspecific inflammation in 8 cases, and showed necrotizing granulomatous inflammation in 2 cases.

Mycobacterial culture was performed on 32 surgical specimens (80\%). Positive results were obtained for 19 patients (47.5\%) and Mycobacterium avium was the most frequently cultured mycobacteria (13 cases, 68\%). All microbiologic culture results were negative for Bartonella species. Positive AFB stains on microbiological examinations were found in 9 patients (23\%). PCR was positive for NTM in 3 patients and for $B$. henselae in 2 patients.

\section{Definitive diagnosis}

The diagnosis of NTM was based on positive cultures only $(n=5,20 \%)$, on positive tuberculin skin test only $(n=4$, $16 \%$ ), on positive AFB stains on microbiological examination only $(n=2,8 \%)$, or on a positive result in more than one of these diagnostic procedure $(n=14,56 \%)$. Positive PCR assays for NTM alone never enabled a definitive diagnosis to be made.

In the CSD group, all six children had a high IgG antibody titer and only one underwent TST with no reaction.

The nine patients in the US group were poorly investigated and results were inconclusive; only three had a TST. Mycobacterial culture was performed in five patients and $\mathrm{IgG}$ antibodies against $B$. henselae were measured in seven.

\section{Discussion}

Cervical granulomatous lymphadenopathies in children can be of non-infectious or infectious origin. CSD and NTM infections are the most frequent infectious etiologies. We performed a case series of 40 children with cervicofacial granulomatous lymphadenitis treated with surgery between 2000 and 2016. We compared clinical characteristics of patients based on final diagnosis and reviewed early and late outcome after surgery.

NTML was the most frequent diagnosis followed by CSD. A group of patients remained without a clear final diagnosis. Demographic or clinical characteristics did not differ between the groups of patients. Children presented with a painless unilateral adenopathy, typically in the submandibular or preauricular areas in the absence of general symptoms.

Diagnosis of these infections is frequently presumptive based on clinical and demographic features. In many cases, laboratory confirmation is not established [8]. Costeffectiveness and lack of irradiation make ultrasonography a helpful imaging tool for diagnosis and follow-up of cervicofacial masse in children. Conversely, CT and IRM rarely change the diagnosis or the surgical approach and none of these imaging modalities show specific findings for NTML or CSDL. Chest X-rays should only be performed in patients with suspicious of tuberculosis or malignancy.

As initial laboratory evaluation we suggest the following analysis: complete blood count, C-reactive protein (CRP), liver enzymes, renal function and LDH (lactate dehydrogenase), in addition to serologic testing for EBV, CMV, HIV, toxoplasmosis and $B$. henselae.

The sensitivity and specificity of serological testing for $B$. henselae are poor. While high IgG titers associated with typical clinical presentations strongly suggest the diagnosis of CSD [12], negative results do not exclude the presence of acute disease. Indeed, some patients never have a detectable antibody response but a serologic follow-up may be helpful to confirm the diagnosis [13].

Tuberculin skin test (TST) is an important diagnosis tool to screen Mycobacterium tuberculosis infection since many years. Nontuberculous mycobacteria infection and BCG vaccination are other causes of positive TST. Sensitivity and specificity of $70 \%$ and $98 \%$, respectively, have been reported for TST in children with proven NTML with an optimal cutoff for a positive test at $5 \mathrm{~mm}$ [9]. In children with $>5 \mathrm{~mm}$ of induration and risk factors for $M$. tuberculosis, interferongamma release assays (IGRAs), an in vitro blood test of cellmediated immune response, may help to distinguish these two entities [10]. This test shows high diagnostic value for TB even if BCG vaccination has been administrated. It can also be used to exclude TB in patients with NTML [11].

Anyhow in case of diagnostic uncertainty or absence of regression of a neck mass, diagnostic and/or therapeutic lymph node excision have to be considered.

Numerous reports have been published during the past 2 decades regarding the management of NTLM. Although complete surgical excision of the enlarged lymph nodes has long been considered the treatment of choice, recent studies have reported successful use of clarithromycin and/or rifabutin antibiotics therapy or wait-and-see approach [14]. Only one randomized controlled trial compared the effectiveness and side effects of surgical excision and conservative antibiotic therapy for NTML. Higher success rates were reported for surgical excision (96\%) compared to antibiotic therapy (66\%) [15]. Esthetic outcome, evaluated at the 1-year followup visit, was significantly better in patients treated by surgery than in with patients treated with antibiotics alone [16].

The recommended surgical procedure for treating infectious cervical lymphadenitis remains debatable. In the present study, we observed higher healing rates and less recurrence after primary complete excision compared to less complete procedures. Our results are in accordance with the conclusions of a recent meta-analysis where the highest 
cure rates $(88.5 \%)$ in NTML were documented after complete excision when compared to other treatment options including incision/drainage, curettage, FNA and incomplete excision [8]. Unfortunately, long-term follow-up data of these patients are lacking. In a prospective study of 105 cases of NTML, Wolinsky reported recurrences ranging from 2 months to 7 years after primary excision [5]. With a median follow-up of 5.8 years in our study, we observed recurrence up to 25 months after primary incision/drainage.

Parker found no significant impact factors for postoperative complications, such as facial nerve dysfunction, recurrence, wound infection and poor scarring [17]. Peripheral facial nerve paresis or paralysis represents a major risk of surgery, especially for lesions located on the submandibular pedicle or on the parotid space [18]. Spinal accessory nerve damage after resection of high jugular nodes has also been reported [19], but it is uncommon. In our study group, we report $20 \%$ of postoperative facial nerve palsy immediately after surgery. However, full recovery was observed after a few weeks in all except two patients. Even if the complication rate is of $5 \%$, several arguments support a surgical approach.

Due to the poor sensitivity and specificity of available non-invasive tests, histological and bacteriological analysis of the surgical specimen are useful to ensure the diagnosis. Furthermore, concurrent infectious, benign and malignant disease may occur [20]. In a prospective study including 721 patients with primarily unclear neck masses, 22 children with seropositive $B$. henselae biology had a final diagnosis of malignant disease on surgical specimens [13]. Fine-needle aspiration and cytology is an alternative to surgery but is difficult to perform in young patients without sedation and rarely shows characteristic histologic features.

Even if the lymph node stage does not affect the success rate of healing [15], surgery performed in an early stage of the disease, before nodes become fluctuant and before fistula formation, results in an easier procedure with less morbidity [21, 22] and the most satisfactory healing rates [5]. In fact, more advanced lymph node disease with extracapsular suppuration tends to increase the difficulty of surgery because anatomical structures like the facial nerve branches are more difficult to individualize. Furthermore, if the skin is involved or in cases of fistula, excision of large skin areas has to be performed, which leads to poorer esthetic results [23]. Unfortunately, the majority of children included in this study had late stage disease with the median duration of symptoms before surgery being 2.7 months, which is comparably longer than in other reported series [5, 23, 24].

Curettage has been proposed as an acceptable alternative to complete excision in children with a more advanced stage of disease [25].

Finally, we would expect the incidence of facial nerve palsy to be reduced by the use of intraoperative facial nerve monitoring. The latter has resulted in objectively demonstrable improvement in facial nerve outcome especially for patients undergoing parotidectomy [26], otologic and neurotologic surgery [27]. To the best of our knowledge, the use of this technique is uncommonly reported in cervicofacial lymph node excision but was implemented in our centers over last 2 years of the inclusion process and showed promising results.

The benefit of using postoperative anti-mycobacterial antibiotic treatment in addition to surgical excision remains uncertain [8]. A few reports have described the successful use of antibiotics for the treatment of NTML following various surgical procedures [28] but this has never been evaluated after surgery in CSDL. Among the patients who underwent primary complete excision in the present study, post-surgery antibiotic therapy was administrated in only nine patients (33\%). No recurrence occurred in the patients without postoperative medical treatment. This is in accordance with the American Infectious Disease Society who do not recommend postoperative antibiotic therapy after surgical excision for treatment of NTML [29].

Management of CSD is more often conservative, based on antibiotic treatment. Significant reduction of the lymph node size in CSD after a 5-day treatment regimen with oral azithromycin versus placebo has been described, but only if treatment is given within the first 30 days of illness [30].

Our study has the typical limitations of a retrospective review including: lack of standardization of diagnosis procedures, treatment strategies, and heterogeneity of data recorded and available for review. Long-term follow-up data were gathered by phone which could be a source of subjectivity. Patient selection is biased by the fact that the patients typically examined in our tertiary centers were patients in advanced stages whom initial diagnosis had been missed and/or in whom conservative treatment had failed.

In conclusion, we confirm good clinical and functional long-term outcome in patients with cervicofacial granulomatous lymphadenitis treated with surgery. We advocate early surgical intervention with complete excision to accelerate healing with acceptable esthetic results. This surgical procedure is both therapeutic and diagnostic to rule out other concomitant disease such as tuberculosis, lymphoma or other malignancies.

Acknowledgements We thank Dr. Mariana Andrade who provided writing and editorial assistance. No commercial funding was received for this purpose.

\section{Compliance with ethical standards}

Conflict of interest The authors declare that they have no conflict of interest. 
Ethical approval This study was approved by the Medical Ethics Committees of St Luc University Hospital, Brussels (ID: 2014-252) and St Pierre University Hospital, Brussels (ID: AK/16-06-89/4684).

\section{References}

1. Knight PJ, Mulne AF, Vassy LE (1982) When is lymph node biopsy indicated in children with enlarged peripheral nodes? Pediatrics 69(4):391-396

2. Adams DO (1976) The granulomatous inflammatory response. A review. Am J Pathol 84(1):164-192

3. Lindeboom JA, Schreuder WH (2016) Similar presentation of cervical lymphadenitis of different etiology in two siblings. Oral Surg Oral Med Oral Pathol Oral Radiol 122(2):51-54

4. Hazra R, Robson CD, Perez-Atayde AR et al (1999) Lymphadenitis due to nontuberculous mycobacteria in children: presentation and response to therapy. Clin Infect Dis 28(1):123-129

5. Wolinsky E (1995) Mycobacterial lymphadenitis in children: a prospective study of 105 nontuberculous cases with long-term follow-up. Clin Infect Dis 20(4):954-963

6. Ridder GJ, Boedeker CC, Technau-Ihling K et al (2002) Role of cat-scratch disease in lymphadenopathy in the head and neck. Clin Infect Dis 35(6):643-649

7. Wolinsky E (1979) Nontuberculous mycobacteria and associated diseases. Am Rev Respir Dis 119(1):107-159

8. Zimmermann P, Tebruegge M, Curtis N et al (2015) The management of non-tuberculous cervicofacial lymphadenitis in children: a systematic review and meta-analysis. J Infect 71(1):9-18

9. Lindeboom JA, Kuijper EJ, Prins JM et al (2006) Tuberculin skin testing is useful in the screening for nontuberculous mycobacterial cervicofacial lymphadenitis in children. Clin Infect Dis 43(12):1547-1551

10. Blyth CC, Best EJ, Jones CA et al (2009) Nontuberculous mycobacterial infection in children: a prospective national study. Pediatr Infect Dis J 28(9):801-805

11. Detjen AK, Keil T, Roll S et al (2007) Interferon-gamma release assays improve the diagnosis of tuberculosis and nontuberculous mycobacterial disease in children in a country with a low incidence of tuberculosis. Clin Infect Dis 45(3):322-328

12. Demers DM, Bass JW, Vincent JM et al (1995) Cat-scratch disease in Hawaii: etiology and seroepidemiology. J Pediatr 127(1):23-26

13. Ridder GJ, Boedeker CC, Technau-Ihling K et al (2005) Catscratch disease: otolaryngologic manifestations and management. Otolaryngol Head Neck Surg 132(3):353-358

14. Lindeboom JA (2011) Conservative wait-and-see therapy versus antibiotic treatment for nontuberculous mycobacterial cervicofacial lymphadenitis in children. Clin Infect Dis 52(2):180-184

15. Lindeboom JA, Kuijper EJ, Bruijnesteijn van Coppenraet ES et al (2007) Surgical excision versus antibiotic treatment for nontuberculous mycobacterial cervicofacial lymphadenitis in children: a multicenter, randomized, controlled trial. Clin Infect Dis 44(8):1057-1064

16. Lindeboom JA, Lindeboom R, Bruijnesteijn van Coppenraet ES et al (2009) Esthetic outcome of surgical excision versus antibiotic therapy for nontuberculous mycobacterial cervicofacial lymphadenitis in children. Pediatr Infect Dis J 28(11):1028-1030

17. Parker NP, Scott AR, Finkelstein M et al (2012) Predicting surgical outcomes in pediatric cervicofacial nontuberculous mycobacterial lymphadenitis. Ann Otol Rhinol Laryngol 121(7):478-484

18. Mahadevan M, Neeff M, Van Der Meer G et al (2016) Non-tuberculous mycobacterial head and neck infections in children: analysis of results and complications for various treatment modalities. Int J Pediatr Otorhinolaryngol 82:102-106

19. Rahal A, Abela A, Arcand PH et al (2011) Nontuberculous mycobacterial adenitis of the head and neck in children: experience from a tertiary care pediatric center. Laryngoscope 111(10):1791-1796

20. Rolain JM, Lepidi H, Zanaret M et al (2006) Lymph node biopsy specimens and diagnosis of cat-scratch disease. Emerg Infect Dis 12(9):1338-1344

21. Pransky SM, Reisman BK, Kearns DB et al (1990) Cervicofacial mycobacterial adenitis in children: endemic to San Diego? Laryngoscope 100(9):920-925

22. Benson-Mitchell R, Buchanan G (1996) Cervical lymphadenopathy secondary to atypical mycobacteria in children. J Laryngol Otol 110:48-51

23. Flint D, Mahadevan M, Barber C et al (2000) Cervical lymphadenitis due to non-tuberculous mycobacteria: surgical treatment and review. Int J Pediatr Otorhinolaryngol 53(3):187-194

24. Thoon KC, Subramania H, Chong CY et al (2014) Granulomatous cervicofacial lymphadenitis in children a nine-year study in Singapore. Singapore Med J 55(8):427-431

25. De Baerdemaeker H, Meertens B (2018) Lymphadenopathy caused by nontuberculous mycobacteriae in children: treatment and outcome [abstract]. In: Proceedings of the 46th Annual Congress of the Belgian Society of Paediatrics (BVK-SBP, Abstract I01)

26. Sood AJ, Houlton JJ, Nguyen SA, Gillespie MB (2015) Facial nerve monitoring during parotidectomy: a systematic review and meta-analysis. Otolaryngol Neck Surg 152:631-637

27. Gidley P, Maw J, Gantz B et al (2018) Contemporary opinions on intraoperative facial nerve monitoring. OTO Open 2(3):1-7

28. Coulter JB, Lloyd DA, Jones M, Cooper JC et al (2006) Nontuberculous mycobacterial adenitis: effectiveness of chemotherapy following incomplete excision. Acta Paediatr 95(2):182-188

29. Griffith DE, Aksamit T, Brown-Elliott BA et al (2017) ATS Mycobacterial Diseases Subcommittee; American Thoracic Society; Infectious Disease Society of America. An official ATS/ IDSA statement: diagnosis, treatment, and prevention of nontuberculous mycobacterial diseases. Am J Respir Crit Care Med 175(4):367-416

30. Bass JW, Freitas BC, Freitas AD et al (1998) Prospective randomized double blind placebo controlled evaluation of azithromycin for treatment of cat-scratch disease. Pediatr Infect Dis J 17(6):447-452

Publisher's Note Springer Nature remains neutral with regard to jurisdictional claims in published maps and institutional affiliations. 